\section{Acknowledgements}

I would like to thank Dr Vijayaprasad Gopichandran, Dr Amar Jesani, Dr Rakhi Ghoshal, Dr P Manickam and Dr Ravi Prasad Verma, for their comments during the Workshop on Public Health Ethics Case Studies held from March 26-27, 2018, at the Achutha Menon Centre for Health Science Studies Thiruvananthapuram.

\section{Notes}

1 In this study, a "couple with infertility" has been defined as a couple who have been married for a minimum of one year, and are seeking treatment, or have a history of treatment, for achieving pregnancy.

2 ASHAs are trained female community health activists. Selected from the community itself and accountable to it, the ASHA is trained to be an interface between the community and the public health system.
3 The Anganwadi or "courtyard shelter" is the focal point for delivery of the government's Integrated Child Development Services, through which children under the age of 6 are given supplementary nutrition, immunisation and pre-school education, and mothers are given supplementary nutrition.

\section{References}

1. Dickson-Swift V, James E L, Kippen S, Liamputtong P. Doing sensitive research: what challenges do qualitative researchers face? Qual Res. 2007.7(3): 327-53.

2. de Laine M. Fieldwork, participation and practice: ethics and dilemmas in qualitative research. London, UK: Sage Publications; 2000. pp 123-6.

3. McAreavey R, Das C. A delicate balancing act: Negotiating with gatekeepers for ethical research when researching minority communities. Int J Qual Methods. 2013 Feb 1. 12(1): 113-131

\title{
Community gatekeepers and the conundrum of confidentiality and coercion
}

\section{VIJAYAPRASAD GOPICHANDRAN}

Keywords: Gatekeepers, public health research, access, coercion, confidentiality,

Sunu Thomas has described (1) a research study in which she gained access through a community health worker to members of the community seeking treatment for infertility. One of the research participants who had undergone such a sensitive treatment and her family were concerned that the community health worker's presence during the interview would breach the confidentiality of their health information. In this commentary, I will discuss the issues of balancing accessibility versus confidentiality, and of coercion in the process of approaching community members through gatekeepers of the community.

Frontline community health workers are often the gatekeepers through whom public health researchers access community members to conduct their research. The health workers are usually themselves members of the community and so have first-hand information about the potential research participants. In a typical rural context in India, public hospital records and health system information may not be reliable, making access to patients difficult. Moreover, even if a researcher got the address details of a potential research participant through hospital records, the complex layout of streets, lanes and houses in rural India makes locating people arduous. Therefore, public health researchers largely depend on community health workers to identify potential research

\footnotetext{
Author: Vijayaprasad Gopichandran (vijay.gopichandran@gmail.com), Assistant Professor, Department of Community Medicine, ESIC Medical College and PGIMSR, KK Nagar, Chennai 600078 INDIA

To cite: Gopichandran V. Community gatekeepers and the conundrum of confidentiality and coercion. Indian J Med Ethics. 2020 Jan-Mar;5(1) NS:11-3. DOI: 10.20529/IJME.2020.009

Peer Reviewer: Sangeeta Rege

CIndian Journal of Medical Ethics 2020
}

participants.

The issue described by the researcher in this case is very common in public health research, especially when it is based in the community. Two years ago, I was involved in a community-based qualitative research project to understand the experiences of women who suffered stillbirths in the public health facilities of Tamil Nadu. Our team approached, through the village health nurses (VHN), the families of women who had suffered a stillbirth. The VHN is the frontline community health worker in Tamil Nadu who takes care of a population of about 5000 individuals and plays the vital role of a bridge between the community and the primary healthcare system. Many of the mothers whom we approached had lost their babies during delivery in the public health system and so had a sense of anger and resentment against the health system. Some of them were even angry with the VHN, as she is the face of the public health system in the community. As described by the author, we too had been worried that the presence of the VHN during the interviews could breach the interviewees' confidentiality.

Multiple ethical concerns have been raised in this case study. I would like to discuss two main confidentiality concerns. The first is whether it is appropriate for researchers to gain access to communities through frontline health workers. Frontline health workers are the vital link between communities and the health system. The community members sometimes reveal very sensitive information about their health to community health workers, in order to secure their help to access health system facilities. Often, the community health worker is part of the community herself. So, being an insider, she is already privy to some personal information about the members and the community. Therefore, like the fiduciary doctor-patient relationship and its inherent covenant of confidentiality, the community health worker's- relationship with the community 
must also be bound by the confidentiality clause. The trust in the community health worker-community relationship is strongly influenced by this confidentiality (2). Therefore, when community health workers are used to reach members of the community, mistrust towards them may develop among the community members (3). Sometimes, the illness under research is stigmatising, such as tuberculosis, sexually transmitted illnesses, infertility etc, and when such sensitive information is shared with researchers, it leads to a breach of trust. We have seen this happen often, but it is never mentioned or discussed because of the power imbalance between community health workers, who are representatives of the health system, and community members who are the beneficiaries. For example, in Tamil Nadu, the VHN is the controller of the Dr Muthulakshmi Reddy Maternity Benefit Scheme and many such welfare schemes, and the community would not want to alienate her, lest they face difficulty in availing of these welfare benefits. Therefore, utilising the intimate knowledge of a community health worker about illnesses in the community for research recruitment can be unethical and can erode trust in the community- health worker relationship.

Before utilising community health workers to approach research participants, the researcher must implement active community engagement. Community members must be notified of the research study and told that potential research participants will be accessed through the community health worker. The community health worker must be encouraged to first seek the permission of potential research participants to share their details with the researchers. This step helps preserve trust in the community health worker, as also the confidence that adequate measures will be taken to protect the confidentiality of patient information.

Secondly, the presence of the community health worker at the data collection site can violate the privacy and confidentiality of participants regarding sensitive health information. In the case described here, not only did the researcher contact the couple with infertility through the community health worker, she also conducted the interview with the health worker close by. In low resource settings in villages in India, it is challenging to secure a private space for conducting qualitative in-depth interviews in the community. Moreover, if the community health worker is accompanying the researcher to help identify the house, it may be difficult to ask the worker to leave the place during the interview. Not only might this be viewed as rude, it may be unsafe in some places to keep the health worker standing outside the house or send her elsewhere.

In our study of the experiences of women who suffered stillbirths, we adopted a few strategies to overcome this difficulty. Wherever possible, we tried to get the address and landmark details of the mother from the VHN and went to her house on our own. Wherever taking the VHN with us was unavoidable, we attempted to interview the mother in private When this was also not possible, we did not delve much into sensitive information during the interview, but established follow-up contacts later over the phone and asked for such information in private. It would always be good practice to emphasise the risks and benefits of participating in qualitative interviews while obtaining informed consent from the participants. We made sure that we told those we interviewed that they may have to recall and relive some painful and traumatic experiences of delivering a still-born child. We also explained that we may request some sensitive information, and if they felt uncomfortable sharing it in the team's presence, they need not. We made sure that both the mother and the health worker were comfortable and felt respected and valued during the process of the interview.

Sometimes accessing research participants through community health workers can end up as coercion of the potential participants. As mentioned earlier, the VHN in rural Tamil Nadu wields significant power and controls the delivery of several important welfare schemes. Therefore, when the VHN brings a researcher to the house of a potential research participant, they may feel obliged to agree to participate. We have found that making it clear that the study is purely voluntary helps the VHN understand that there is no pressure from the higher up authorities to participate in the study. Many health workers are used to working under pressure to meet targets, for example, counselling at least 10 mothers a year to accept sterilisation surgeries, bringing all eligible children in for vaccination etc. Sometimes they are incentivised for meeting targets, and even penalised for not meeting them. Therefore, they tend to think of identifying potential research participants as one of their many health-related targets and to adopt coercive measures to get people to participate. To deal with this problem, we have found it very useful to declare upfront that the research is not part of the concerned worker's health system function, and to clarify that identifying research participants for us will not impact their performance, incentives, appraisal or career, in any way. This greatly helps reduce coercion. In one of our studies, after initial experiences of possible coercion of research participants, we modified our recruitment strategy. We requested the VHN to convey the information about the study to the potential participants and get permission for us to contact them. From that point onwards, the research team took over the entire recruitment, consent, interview and reporting formalities, leaving the VHN out of the picture to avoid any tinge of coercion.

One other potential solution to this conundrum is to train community health workers as actual research investigators, rather than treating them as just access points to the participants. They may be trained on issues of research ethics such as upholding the autonomy of the participants, maintaining privacy and confidentiality of research information and ensuring justice. This will not only empower the public health research system; it will also ensure the ethical conduct of research. Once community health workers are inducted as part of the public health research team, they may also be requested to enter into contractual confidentiality agreements as part of the research. This adds a further layer of confidentiality protection for research participants. 
It is very important to gain access to communities through trusted community health workers. Not only does this provide credibility to the researcher, it also creates a level of comfort for the research participant to be actively involved in the interview or discussion during data collection. However, key issues of confidentiality and potential coercion of the research participants are both very important ethical considerations in the design and conduct of community-based public health research (4). These issues must be considered and discussed by the researcher at the design stage and subsequently by the ethics committee during ethics review of the research proposal.

\section{References}

1. Thomas S. Maintaining confidentiality while gaining access to the community, Indian J Med Ethics. 2020 Jan-Mar;5(1) NS:10-1. DOI: 10.20529/IJME.2020.008

2. Geldsetzer P, Vaikath M, De Neve JW, Bossert TJ, Sibandze S, Mkhwanazi M, Bärnighausen T. Distrusting community health workers with confidential health information: a convergent mixed-methods study in Swaziland. Health Policy Plan 2017 Jul 1;32(6):882-9.

3. Grant M, Wilford A, Haskins L, Phakathi S, Mntambo N, Horwood CM. Trust of community health workers influences the acceptance of community-based maternal and child health services. Afr J Prim Health Care Fam Med. 2017 May 29;9(1): 1281.

4. Damianakis T, Woodford M.R. Qualitative research with small connected communities: generating new knowledge while upholding research ethics. Qual Health Res. 2012 May 22; 22(5):708-18.

\section{Autonomy and risk in health policy and systems research}

\section{MALU MOHAN}

Keywords: Autonomy, risk, health policy and systems research, informed consent, written consent, waiver of consent, participation in HPSR

\section{Introduction and context}

Health policy and systems research (HPSR), a critical area of public health research, aims to enhance our understanding about how health systems function and how health policies are generated and implemented. HPSR predominantly operates in "real life contexts" of communities or institutions (1). Such contexts significantly reduce the ability of researchers to exert the same level of control over their environment that is possible in biomedical/clinical research. A recent expert consultation led by the World Health Organisation, to explore the knowledge gap relevant to ethical review and practice of HPSR, recommends capacity building for local research ethics committees to deal with its complexities (2). This case presents the ethical dilemma faced by a public health researcher exploring the development of key policies governing the regulation of a specific branch of the health professions.

\section{The case}

The research aimed to analyse the policies which governed the education sector of a particular branch of health professional education in the country. This required interviewing civil servants and other officials from central regulatory agencies. The Institutional Ethics Committee (IEC) of the central institute

\footnotetext{
Author: Malu Mohan (dr.malumohan@gmail.com), Research Coordinator Project on Decentralisation and Health: Lessons from the Kerala Experience, Kerala Institute of Local Administration, Thrissur, Kerala, INDIA.

To cite: Mohan M. Autonomy and risk in health policy and systems research. Indian J Med Ethics. 2020 Jan-Mar;5(1) NS:13-4 DOI: 10.20529/IJME.2020.010.

CIndian Journal of Medical Ethics 2020.
}

where this research was conceived follows the Indian Council for Medical Research guidelines for ethical review of research proposals. These guidelines are predominantly governed by a biomedical/clinical research paradigm. The guidelines mandate that researchers obtain written informed consent from all prospective participants or their legally acceptable/authorised representatives, with few specific circumstances where a waiver could be sought. The researcher anticipated that most of the policy actors would not be comfortable signing a written informed consent document. However, the IEC directed that all participants had to give written informed consent prior to participation.

After the research proposal was cleared by the IEC, a key actor in the policy process was introduced to the researcher. This was a central government officer who had served for a long period in one of the central regulatory agencies responsible for major policy decisions relevant to the research. He told the researcher that he disagreed with many of these policy decisions but did not oppose them because he was just a cog in the wheel and had limited ability to initiate pressures or resist change.

The officer expressed willingness to participate in the study and share his own notes and personal research. He also consented to audio recording of the interviews on condition that he would operate the recorder himself, so that he could pause the recording in the midst of a conversation when he did not want to place the divulging of a specific piece of information on record, and continue when he wished to be on record. However, when the researcher sought his written informed consent to participate, the officer categorically stated that although he was willing to cooperate with the research, there was no question of signing any document.

\section{The dilemma}

The retired official could be a key informant to the research, 